\title{
On Singularity Formation of a Nonlinear Nonlocal System
}

\author{
Thomas Y. Hou* Congming $\mathrm{Li}^{\dagger} \quad$ Zuoqiang $\mathrm{Shi}^{\ddagger} \quad \mathrm{Shu}$ Wang§ ${ }^{\S} \quad \mathrm{Xinwei} \mathrm{Yu}^{\Uparrow}$
}

December 12, 2013

\begin{abstract}
We investigate the singularity formation of a nonlinear nonlocal system. This nonlocal system is a simplified one-dimensional system of the 3D model that was recently proposed by Hou and Lei in [16] for axisymmetric 3D incompressible Navier-Stokes equations with swirl. The main difference between the 3D model of Hou and Lei and the reformulated 3D Navier-Stokes equations is that the convection term is neglected in the 3D model. In the nonlocal system we consider in this paper, we replace the Riesz operator in the 3D model by the Hilbert transform. One of the main results of this paper is that we prove rigorously the finite time singularity formation of the nonlocal system for a large class of smooth initial data with finite energy. We also prove the global regularity for a class of smooth initial data. Numerical results will be presented to demonstrate the asymptotically self-similar blow-up of the solution. The blowup rate of the self-similar singularity of the nonlocal system is similar to that of the 3D model.
\end{abstract}

Key words: Finite time singularities, nonlinear nonlocal system, stabilizing effect of convection.

\section{Introduction}

The question of whether a solution of the 3D incompressible Navier-Stokes equations can develop a finite time singularity from smooth initial data with finite energy is one of the most outstanding mathematical open problems [11, 20, 23]. A main difficulty in obtaining the global regularity of the 3D Navier-Stokes equations is due to the presence of the vortex stretching term, which has a formal quadratic nonlinearity in vorticity. So far, most regularity analysis for the 3D Navier-Stokes equations uses energy estimates. Due to the incompressibility condition, the convection term does not contribute to the energy norm

\footnotetext{
*Applied and Comput. Math, Caltech, Pasadena, CA 91125. Email: hou@acm.caltech.edu.

${ }^{\dagger}$ Department of Applied Mathematics, University of Colorado, Boulder, CO. 80309. Email: cli@colorado.edu

${ }^{\ddagger}$ Applied and Comput. Math, Caltech, Pasadena, CA 91125. Email: shi@acm.caltech.edu.

${ }^{\S}$ College of Applied Sciences, Beijing University of Technology, Beijing 100124, China. Email: wangshu@bjut.edu.cn

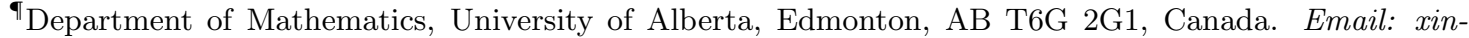
weiyu@math.ualberta.ca
} 
of the velocity field or any $L^{p}(1<p \leq \infty)$ norm of the vorticity field. In a recent paper by Hou and Lei [16], the authors investigated the stabilizing effect of convection by constructing a new 3D model for axisymmetric 3D incompressible Navier-Stokes equations with swirl. This model preserves almost all the properties of the full 3D Navier-Stokes equations except for the convection term which is neglected. If one adds the convection term back to the 3D model, one would recover the full Navier-Stokes equations. They also presented numerical evidence which supports that the 3D model may develop a potential finite time singularity. They further studied the mechanism that leads to these singular events in the 3D model and how the convection term in the full Navier-Stokes equations destroys such a mechanism.

In this paper, we propose a simplified nonlocal system for the 3D model proposed by Hou and Lei in [16]. The nonlocal system is derived by first reformulating the 3D model of Hou and Lei as the following two-by-two nonlinear and nonlocal system of partial differential equations:

$$
u_{t}=2 u v+\nu \Delta u, \quad v_{t}=(-\Delta)^{-1} \partial_{z z} u^{2}+\nu \Delta v,
$$

where $u=u^{\theta} / r, v=\psi_{z}^{\theta} / r$, and $\Delta=\partial_{z}^{2}+\partial_{r}^{2}+\frac{3}{r} \partial_{r}$, and $u^{\theta}$ is the angular velocity component and $\psi^{\theta}$ is the angular stream function respectively, $r=\sqrt{x^{2}+y^{2}}$. By the partial regularity result for the 3D model [14], which is an analogue of the well-known Caffarelli-Kohn-Nirenberg partial regularity theory for the 3D incompressible Navier-Stokes equations [2], we know that the singularity can only occur along the symmetry axis, i.e. the $z$-axis. In order to study the potential singularity formation of the $3 \mathrm{D}$ model, it makes sense to construct a simplified one dimensional nonlocal system along the $z$-axis. One obvious choice is to replace the Riesz operator $(-\Delta)^{-1} \partial_{z}^{2}$ by the Hilbert transform $H$ along the $z$ axis, and replace $\Delta u$ by $u_{z z}, \Delta v$ by $v_{z z}$. This gives rise to our simplified nonlocal system:

$$
u_{t}=2 u v+\nu u_{z z}, \quad v_{t}=H\left(u^{2}\right)+\nu v_{z z}
$$

where $H$ is the Hilbert transform,

$$
(H f)(x)=\frac{1}{\pi} \mathrm{P} \cdot \mathrm{V} \cdot \int_{-\infty}^{\infty} \frac{f(y)}{x-y} \mathrm{~d} y .
$$

In our analysis, we will focus on the inviscid version of the nonlocal system and relabel the variable $z$ as $x$ :

$$
u_{t}=2 u v, \quad v_{t}=H\left(u^{2}\right)
$$

with the initial condition

$$
u(t=0)=u_{0}(x), \quad v(t=0)=v_{0}(x) .
$$

Note that the 1D model (2) is designed to capture the dynamics of the 3D model (1) along the $z$-axis only. Thus, its inviscid model (4) does not enjoy the energy conservation property of the original model in the three-dimensional space.

One of the main results of this paper is that we prove rigorously the finite time singularity formation of the nonlocal system for a large class of smooth initial data with finite energy. 
As we will demonstrate in this paper, the blowup rate of the self-similar singularity of the nonlocal system (4)-(5) is qualitatively similar to that of the 3D model. The main result of this paper is summarized in the following theorem.

Theorem 1.1 Assume that the support of $u_{0}$ is contained in $(a, b)$ and that $u_{0}, v_{0} \in H^{1}$. Let $\phi(x)=x-a$ and

$$
C=4 \int_{a}^{b} \phi(x) u_{0}^{2} v_{0} \mathrm{~d} x, \quad I_{\infty}=\int_{0}^{+\infty} \frac{\mathrm{d} y}{\sqrt{y^{3}+1}} .
$$

If $C>0$, then the solution of the nonlocal system (4)-(5) must develop a finite time singularity in the $H^{1}$ norm no later than $T^{*}=\left(\frac{4 C}{3 \pi(b-a)^{2}}\right)^{-1 / 3} I_{\infty}$.

A similar result has been obtained for periodic initial data.

The analysis of the finite time singularity for this nonlocal system is rather subtle. The main technical difficulty is that this is a two-by-two nonlinear nonlocal system. The key issue is under what condition the solution $u$ has a strong alignment with the solution $v$ dynamically. If $u$ and $v$ have a strong alignment for long enough time, then the right hand side of the $u$ equation would develop a quadratic nonlinearity dynamically, which will lead to a finite time blowup. Note that $v$ is coupled to $u$ in a nonlinear and nonlocal fashion through the Hilbert transform. It is not clear whether $u$ and $v$ will develop such a nonlinear alignment dynamically. To establish such a nonlinear alignment, we need to use the following important property of the Hilbert transform:

Proposition 1.1 Let $\phi$ be a globally Lipschitz continuous function on $R$. For any $f \in$ $L^{p}\left(R^{1}\right) \cap L^{1}\left(R^{1}\right)$ and $\phi f \in L^{q}\left(R^{1}\right)$ with $\frac{1}{p}+\frac{1}{q}=1,1<p, q<+\infty$, we have

$$
\int_{-\infty}^{+\infty} \phi(x) f(x) H f(x) \mathrm{d} x=\frac{1}{2 \pi} \int_{-\infty}^{+\infty} \int_{-\infty}^{+\infty} \frac{\phi(x)-\phi(y)}{x-y} f(x) f(y) \mathrm{d} x \mathrm{~d} y .
$$

Using this property, we can identify an appropriate test function $\phi$ such that the time derivative of $\int u^{2} \phi d z$ satisfies a nonlinear inequality. This inequality implies a finite time blowup of the nonlocal system.

Proposition 1.1 should be a well-known property in the Harmonic Analysis literature. During the revision of our paper, we found that an identity which can be used to derive the special case $\phi=x$ of Proposition 1.1 has been used in [10], see also a recent paper [19] ${ }^{1}$. However, we have not been able to find a proof for the general case stated in Proposition 1.1 in the literature. For the sake of completeness, we provide a proof of Proposition 1.1 in Section 2.

Another interesting result is that we prove the global regularity of our nonlocal system for a class of smooth initial data. Specifically, we prove the following theorem:

\footnotetext{
${ }^{1}$ We only learned about the work of [19] after the presentation of our work at the PIMS workshop on Hydrodynamics Regularity in August 2009.
} 
Theorem 1.2 Assume that $u_{0}, v_{0} \in H^{1}$. Further we assume that $u_{0}$ has compact support in an interval of size $\delta$ and $v_{0}$ satisfies the condition $v_{0} \leq-3$ on this interval. Then the $H^{1}$ norm of the solution of the nonlocal system (4)-(5) remains bounded for all time as long as the following holds

$$
\delta^{1 / 2}\left(\left\|v_{0 x}\right\|_{L^{2}}+\frac{1}{3} \delta^{1 / 2}\left\|u_{0 x}\right\|_{L^{2}}^{2}\right)<\frac{1}{4} .
$$

Moreover, we have $\|u\|_{L^{\infty}} \leq C e^{-3 t},\|u\|_{H^{1}} \leq C e^{-3 t}$, and $\|v\|_{H^{1}} \leq C$ for some constant $C$ which depends on $u_{0}, v_{0}$, and $\delta$ only.

In order to study the nature of the singularities, we have performed extensive numerical experiments for the nonlocal system with or without viscosity. Our numerical study shows that $\|u\|_{L^{\infty}}(t)$ and $\|v\|_{L^{\infty}}(t)$ develop a finite time blowup with a blowup rate $O\left(\frac{1}{T-t}\right)$, which is qualitatively similar to that of the $3 \mathrm{D}$ model [16]. Our numerical results also indicate that the solution of the inviscid nonlocal system seems to develop a one-parameter family self-similar finite time singularity of the type:

$$
\begin{aligned}
u(x, t) & =\frac{1}{T-t} U(\xi, t) \\
v(x, t) & =\frac{1}{T-t} V(\xi, t) \\
\xi & =\frac{x-x_{0}(t)}{(T-t)^{1 / 2} \log (1 /(T-t))^{1 / 2}}
\end{aligned}
$$

where $x_{0}(t)$ is the position at which $u(x, t)$ achieves its maximum. The parameter that characterizes this self-similar blowup is the rescaled speed of propagation of the traveling wave defined as follows:

$$
\lambda=\lim _{t \rightarrow T}\left((T-t)^{1 / 2} \frac{d}{d t} x_{0}(t)\right) .
$$

Different initial data give different speeds of propagation of the singularity. One of the interesting findings of our numerical study is that by rescaling the self-similar variable $\xi$ by $\lambda^{-1}$, the different rescaled profiles corresponding to different initial conditions all collapse to the same universal profile. We offer some preliminary analysis to explain this phenomenon.

Our numerical results also show that there is a significant overlap between the inner region of $U$ and the inner region of $V$ where $V$ is positive. Such overlap persists dynamically and is responsible for producing a quadratic nonlinearity in the right hand side of the $u$ equation. The nonlinear interaction between $u$ and $v$ produces a traveling wave that moves to the right ${ }^{2}$. Such phenomenon seems quite generic, and is qualitatively similar to that of the 3D model [16]. The only difference is that the 3D model produces traveling waves that move along the symmetry axis in both directions. It is still a mystery why the inviscid nonlocal system selects the scaling (10) with the $1 / 2$ exponent and a logarithmic correction. With the logarithmic correction, the viscous term can not dominate the nonlinear term $2 u v$ in the equation. Indeed, when we add viscosity to the nonlocal system, we find that the

\footnotetext{
${ }^{2}$ If we change the plus sign in front of the Hilbert transform in the nonlocal system (2) to a minus sign, the nonlocal system would produce a traveling wave that moves to the left.
} 
viscous solution still develops the same type self-similar finite time blowup as that of the inviscid nonlocal system.

We remark that Hou, Shi and Wang [17] have recently made some important progress in proving the formation of finite time singularities of the original 3D model of Hou and Lei [16] for a class of smooth initial conditions with finite energy under some appropriate boundary conditions. The stabilizing effect of convection has been studied by Hou and Li in a recent paper [15] via a new 1D model. Formation of singularities for various model equations for the 3D Euler equations or the surface quasi-geostrophic equation has been investigated by Constantin-Lax-Majda [6], Constantin [5], DeGregorio [8, 9], Okamoto and Ohkitani [21], Cordoba-Cordoba-Fontelos [7], Chae-Cordoba-Cordoba-Fontelos [4], and Li-Rodrigo [18].

The rest of the paper is organized as follows. In Section 2, we study some properties of the nonlocal system. In Section 3, we establish the local well-posedness of the nonlocal system. Section 4 is devoted to proving the finite time singularity formation of the inviscid nonlocal system for a large class of smooth initial data with finite energy. We prove the global regularity of the nonlocal system for a class of initial data in Section 5. Finally, we present several numerical results in Section 6 to study the nature of the finite time singularities for both the inviscid and viscous nonlocal systems.

\section{Properties of the nonlocal system}

In this section, we study some properties of the nonlocal system. First of all, we note that the nonlocal system has some interesting scaling property. Specifically, for any constants $\alpha$ and $\beta$ satisfying $\alpha \beta>0$, the nonlocal system

$$
u_{t}=\alpha u v, \quad v_{t}=\beta H u^{2}
$$

is equivalent to the system

$$
\tilde{u}_{t}=2 \tilde{u} \tilde{v}, \quad \tilde{v}_{t}=H \tilde{u}^{2}
$$

by introducing the following rescaling of the solution:

$$
u=\tilde{u}(x, \gamma t), \quad v=\mu \tilde{v}(x, \gamma t),
$$

where $\gamma$ and $\mu$ are related to $\alpha$ and $\beta$ through the following relationship:

$$
\gamma=\sqrt{\frac{\alpha \beta}{2}}, \quad \mu=\operatorname{sgn}(\alpha) \sqrt{\frac{2 \beta}{\alpha}} .
$$

Therefore, it is sufficient to consider the nonlocal system in the following form:

$$
u_{t}=2 u v, \quad v_{t}=H u^{2}
$$

Moreover, if we replace the second equation $v_{t}=H u^{2}$ by $v=H u^{2}$ and define $w=u^{2}$, then our nonlocal system is reduced to the well-known Constantin-Lax-Majda model [6]:

$$
w_{t}=4 w H w
$$


Before we end this section, we present the proof of Proposition 1.1.

Proof of Proposition 1.1. Denote $\widetilde{f}_{\epsilon}(x)=\frac{1}{\pi} \int_{|x-y| \geq \epsilon} \frac{f(y)}{x-y} \mathrm{~d} y, F_{\epsilon}(x)=\phi(x) f(x) \widetilde{f}_{\epsilon}(x)$ and $\bar{f}(x)=\sup _{\epsilon \geq 0}\left|\widetilde{f}_{\epsilon}(x)\right|$. It follows from the singular integral theory of Calderon-Zygmund [3] that $\widetilde{f}_{\epsilon}(x) \rightarrow H f(x)$ a.e. $x \in R^{1}$ and

$$
\|\bar{f}\|_{L^{p}} \leq C_{p}\|f\|_{L^{p}}
$$

Therefore, we have $F_{\epsilon}(x) \rightarrow \phi(x) f(x) H f(x)$ a.e. $\quad x \in R^{1}$ and $\left|F_{\epsilon}(x)\right| \leq G(x)$, where $G(x)=|\phi(x) f(x)| \bar{f}(x)$ satisfies

$$
\begin{aligned}
\|G(x)\|_{L^{1}} & \leq\|\bar{f}(x)\|_{L^{p}}\|\phi(x) f(x)\|_{L^{q}} \\
& \leq C_{p}\|f(x)\|_{L^{p}}\|\phi(x) f(x)\|_{L^{q}}<+\infty .
\end{aligned}
$$

Using the Lebesgue Dominated Convergence Theorem, we have

$$
\begin{aligned}
\int \phi(x) f(x) H(f) \mathrm{d} x & =\lim _{\epsilon \rightarrow 0} \int f(x) \phi(x) \tilde{f}_{\epsilon}(x) d x \\
& =\frac{1}{\pi} \lim _{\epsilon \rightarrow 0} \int f(x) \phi(x) \int_{|x-y| \geq \epsilon} \frac{f(y)}{x-y} \mathrm{~d} y \mathrm{~d} x .
\end{aligned}
$$

Note that

$$
\begin{aligned}
\int|f(y)|\left(\int_{|x-y| \geq \epsilon} \frac{|f(x) \phi(x)|}{|x-y|} \mathrm{d} x\right) \mathrm{d} y & \leq \int|f(y)|\left(\int \frac{2|f(x) \phi(x)|}{\epsilon+|x-y|} \mathrm{d} x\right) \mathrm{d} y \\
& \leq 2\|\phi(x) f(x)\|_{L^{q}}\left\|(\epsilon+|x|)^{-1}\right\|_{L^{p}} \int|f(y)| \mathrm{d} y \\
& =C\|f(y)\|_{L^{1}}\|\phi(x) f(x)\|_{L^{q}}<\infty,
\end{aligned}
$$

for each fixed $\epsilon>0$ since $f \in L^{1}, \phi f \in L^{q}$ by our assumption, and $C \equiv\left\|(\epsilon+|x|)^{-1}\right\|_{L^{p}}<\infty$ for $p>1$. Thus Fubini's Theorem implies that

$$
\frac{1}{\pi} \int f(x) \phi(x) \int_{|x-y| \geq \epsilon} \frac{f(y)}{x-y} \mathrm{~d} y \mathrm{~d} x=\frac{1}{\pi} \iint_{|x-y| \geq \epsilon} f(x) \phi(x) \frac{f(y)}{x-y} \mathrm{~d} y \mathrm{~d} x
$$

for each fixed $\epsilon>0$. Furthermore, by renaming the variables in the integration, we can rewrite $1 / 2$ of the integral on the right hand side of (19) as follows:

$$
\frac{1}{2 \pi} \iint_{|x-y| \geq \epsilon} f(x) f(y) \frac{\phi(x)}{x-y} \mathrm{~d} y \mathrm{~d} x=-\frac{1}{2 \pi} \iint_{|x-y| \geq \epsilon} f(x) f(y) \frac{\phi(y)}{x-y} \mathrm{~d} x \mathrm{~d} y,
$$

which implies that

$$
\frac{1}{\pi} \iint_{|x-y| \geq \epsilon} f(x) f(y) \frac{\phi(x)}{x-y} \mathrm{~d} y \mathrm{~d} x=\frac{1}{2 \pi} \iint_{|x-y| \geq \epsilon} f(x) f(y) \frac{\phi(x)-\phi(y)}{x-y} \mathrm{~d} x \mathrm{~d} y .
$$


Since $f \in L^{1}(R)$ and $\phi(x)$ is globally Lipschitz continuous on $R$, it is easy to show that

$$
f(x) f(y) \frac{\phi(x)-\phi(y)}{x-y} \in L^{1}\left(R^{2}\right) .
$$

Using the Lebesgue Dominated Convergence Theorem, we have

$$
\frac{1}{2 \pi} \lim _{\epsilon \rightarrow 0} \iint_{|x-y| \geq \epsilon} f(x) f(y) \frac{\phi(x)-\phi(y)}{x-y} \mathrm{~d} x \mathrm{~d} y=\frac{1}{2 \pi} \iint f(x) f(y) \frac{\phi(x)-\phi(y)}{x-y} \mathrm{~d} x \mathrm{~d} y .
$$

Proposition 1.1 now follows from (18)-(21).

We remark that Proposition 1.1 is also valid for periodic functions. Recall that for periodic functions (with period $2 \pi$ ) the Hilbert transform takes the form:

$$
(H f)(x)=\frac{1}{2 \pi} \text { P.V. } \int_{0}^{2 \pi} f(y) \cot \left(\frac{x-y}{2}\right) \mathrm{d} y .
$$

For the sake of completeness, we state the corresponding result for periodic functions below:

Proposition 2.1 Let $\phi$ be a periodic Lipschitz continuous function with period $2 \pi$. For any periodic function $f$ with period $2 \pi$ satisfying $f \in L^{p}([0,2 \pi])$ and $\phi f \in L^{q}([0,2 \pi])$ with $\frac{1}{p}+\frac{1}{q}=1,1<p, q<+\infty$, we have

$$
\int_{0}^{2 \pi} \phi(x) f(x) H f(x) \mathrm{d} x=\frac{1}{4 \pi} \int_{0}^{2 \pi} \int_{0}^{2 \pi}(\phi(x)-\phi(y)) \cot \left(\frac{x-y}{2}\right) f(x) f(y) \mathrm{d} x \mathrm{~d} y .
$$

The proof of Proposition 2.1 goes exactly the same as the non-periodic case. We omit the proof Here.

Remark 2.1 As we see in the proof of Proposition 1.1, the key is to use the oddness of the kernel in the Hilbert transform. The same observation is still valid here:

$$
\begin{aligned}
& \frac{1}{2 \pi} \iint_{[0,2 \pi]^{2},|x-y|>\epsilon} f(x) f(y) \phi(x) \cot \left(\frac{x-y}{2}\right) \mathrm{d} y \mathrm{~d} x \\
& =-\frac{1}{2 \pi} \iint_{[0,2 \pi]^{2},|x-y|>\epsilon} f(x) f(y) \phi(y) \cot \left(\frac{x-y}{2}\right) \mathrm{d} x \mathrm{~d} y,
\end{aligned}
$$

by renaming the variables in the integration.

\section{Local well-posedness in $H^{1}$}

In this section, we will establish the local well-posedness in Sobolev space $H^{1}$.

Theorem 3.1 (Local well-posedness) For any $u_{0}, v_{0} \in H^{1}$, there exists a finite time $T=$ $T\left(\left\|u_{0}\right\|_{H^{1}},\left\|v_{0}\right\|_{H^{1}}\right)>0$ such that the nonlocal system (4)-(5) has a unique smooth solution, $u, v \in C^{1}\left([0, T) ; H^{1}\right)$ for $0 \leq t \leq T$. Moreover, if $T$ is the first time at which the solution of the nonlocal system ceases to be regular in $H^{1}$ and $T<\infty$, then the solution must satisfy the following condition:

$$
\int_{0}^{T}\left(\|u\|_{L^{\infty}}+\|v\|_{L^{\infty}}\right) \mathrm{d} t=+\infty .
$$


Remark 3.1 We remark that the condition (24) is an analogue of the well-known BealeKato-Majda blowup criteria for the 3D incompressible Euler equation [1].

Proof To show local well-posedness, we write the system as an ODE in the Banach space $X:=H^{1} \times H^{1}$ :

$$
U_{t}=F(U)
$$

where $U=(u, v), F(U)=\left(2 u v, H\left(u^{2}\right)\right)$. As $H^{1}(\mathbb{R})$ is an algebra, $F$ maps any open set in $X$ into $X$, and furthermore $F$ is locally Lipschitz on $X$. Local well-posedness of (4)-(5) then follows from the standard abstract ODE theory such as Theorem 4.1 in [20].

The blow-up criterion (24) follows from the following a priori estimates. Multiplying the $u$-equation by $u$ and the $v$-equation by $v$, and integrating over $R$, we obtain

$$
\frac{\mathrm{d}}{\mathrm{d} t} \int u^{2} \mathrm{~d} x=4 \int u^{2} v \mathrm{~d} x \leq 4\|v\|_{L^{\infty}} \int u^{2} \mathrm{~d} x
$$

and

$$
\begin{aligned}
\frac{\mathrm{d}}{\mathrm{d} t} \int v^{2} \mathrm{~d} x & =2 \int v H u^{2} \mathrm{~d} x=-2 \int(H v) u^{2} \mathrm{~d} x \leq 2\|u\|_{L^{\infty}} \int|H v| u \mathrm{~d} x \\
& \leq\|u\|_{L^{\infty}}\left(\int u^{2} \mathrm{~d} x+\int v^{2} \mathrm{~d} x\right) .
\end{aligned}
$$

Similarly, we can derive $L^{2}$ estimates for $u_{x}$ and $v_{x}$ as follows:

$$
\begin{aligned}
\frac{\mathrm{d}}{\mathrm{d} t} \int u_{x}^{2} \mathrm{~d} x & =4 \int\left(v u_{x}^{2}+u v_{x} u_{x}\right) \mathrm{d} x \\
& \leq 4\|v\|_{L^{\infty}} \int u_{x}^{2} \mathrm{~d} x+2\|u\|_{L^{\infty}} \int\left(u_{x}^{2}+v_{x}^{2}\right) \mathrm{d} x
\end{aligned}
$$

and

$$
\begin{aligned}
\frac{\mathrm{d}}{\mathrm{d} t} \int v_{x}^{2} \mathrm{~d} x & =4 \int v_{x} H\left(u u_{x}\right) \mathrm{d} x \\
& =4 \int\left(H v_{x}\right) u u_{x} \mathrm{~d} x \\
& \leq 2\|u\|_{L^{\infty}} \int\left(u_{x}^{2}+v_{x}^{2}\right) \mathrm{d} x .
\end{aligned}
$$

Summing up the above estimates gives

$$
\frac{\mathrm{d}}{\mathrm{d} t}\left(\|u\|_{H^{1}}^{2}+\|v\|_{H^{1}}^{2}\right) \leq C\left(\|u\|_{L^{\infty}}+\|v\|_{L^{\infty}}\right)\left(\|u\|_{H^{1}}^{2}+\|v\|_{H^{1}}^{2}\right) .
$$

We see that the regularity is controlled by the quantity

$$
\|u\|_{L^{\infty}}+\|v\|_{L^{\infty}}
$$

If $\int_{0}^{T}\left(\|u\|_{L^{\infty}}+\|v\|_{L^{\infty}}\right) d t<\infty$, then it follows from (30) that $\|u\|_{H^{1}}+\|v\|_{H^{1}}$ must remain finite up to $T$. Therefore, if $T$ is the first time at which the solution blows up in the $H^{1}$-norm, we must have

$$
\int_{0}^{T}\left(\|u\|_{L^{\infty}}+\|v\|_{L^{\infty}}\right) \mathrm{d} t=+\infty
$$




\section{Blow up of the nonlocal system}

In this section, we will prove the main result of this paper, that is the solution of the nonlocal system will develop a finite time singularity for a class of smooth initial conditions with finite energy. We will prove the finite time singularity of the nonlocal system as an initial value problem in the whole space and in a periodic domain.

\subsection{Initial Data with Compact Support}

We first consider the initial value problem in the whole space and prove the finite time blow up of the solution of the nonlocal system (4)-(5) for a large class of initial data $u_{0}$ that have compact support.

For the sake of completeness, we will restate the main result below:

Theorem 4.1 Assume that the support of $u_{0}$ is contained in $(a, b)$ and that $u_{0}, v_{0} \in H^{1}$. Let $\phi(x)=x-a$ and

$$
C=4 \int_{a}^{b} \phi(x) u_{0}^{2} v_{0} \mathrm{~d} x, \quad I_{\infty}=\int_{0}^{+\infty} \frac{\mathrm{d} y}{\sqrt{y^{3}+1}} .
$$

If $C>0$, then the solution of the nonlocal system (4)-(5) must develop a finite time singularity in the $H^{1}$ norm no later than $T^{*}=\left(\frac{4 C}{3 \pi(b-a)^{2}}\right)^{-1 / 3} I_{\infty}$.

Proof By Theorem 3.1, we know that there exists a finite time $T=T\left(\left\|u_{0}\right\|_{H^{1}},\left\|v_{0}\right\|_{H^{1}}\right)>0$ such that the nonlocal system (4)-(5) has a unique smooth solution, $u, v \in C^{1}\left([0, T) ; H^{1}\right)$ for $0 \leq t<T$. Let $T^{*}$ be the largest time such that the nonlocal system with initial condition $u_{0}$ and $v_{0}$ has a smooth solution in $H^{1}$. We claim that $T^{*}<\infty$. We prove this by contradiction.

Suppose that $T^{*}=\infty$, i.e. that the nonlocal system has a globally smooth solution in $H^{1}$ for the given initial condition $u_{0}$ and $v_{0}$. Using (4), we obtain

$$
\left(u^{2}\right)_{t t}=4\left(u^{2} v\right)_{t}=8 u_{t} u v+4 u^{2} v_{t}=4\left(u_{t}\right)^{2}+4 u^{2} H\left(u^{2}\right) .
$$

Multiplying $\phi(x)$ to both sides of the above equation and integrating over $[a, b]$, we have the following estimate:

$$
\begin{aligned}
\frac{d^{2}}{d t^{2}} \int_{a}^{b} \phi(x) u^{2}(x, t) \mathrm{d} x & =4 \int_{a}^{b} \phi(x)\left(u_{t}\right)^{2} \mathrm{~d} x+4 \int_{a}^{b} \phi(x) u^{2} H\left(u^{2}\right) \mathrm{d} x \\
& \geq 4 \int_{a}^{b} \phi(x) u^{2} H\left(u^{2}\right) \mathrm{d} x .
\end{aligned}
$$

Note that the support of $u(x, t)$ is the same as that of the initial value $u_{0}$. Proposition 1.1 implies that

$$
\int_{a}^{b} \phi(x) u^{2} H\left(u^{2}\right) \mathrm{d} x=\int_{-\infty}^{\infty} \phi(x) u^{2} H\left(u^{2}\right) \mathrm{d} x
$$




$$
\begin{aligned}
& =\frac{1}{2 \pi} \int_{-\infty}^{\infty} \int_{-\infty}^{\infty} u^{2}(x, t) u^{2}(y, t) \frac{\phi(x)-\phi(y)}{x-y} \mathrm{~d} x \mathrm{~d} y \\
& =\frac{1}{2 \pi}\left(\int_{a}^{b} u^{2}(x, t) \mathrm{d} x\right)^{2} .
\end{aligned}
$$

Combining (34) with (35), we get

$$
\frac{d^{2}}{d t^{2}} \int_{a}^{b} \phi(x) u^{2}(x, t) \mathrm{d} x \geq \frac{2}{\pi}\left(\int_{a}^{b} u^{2}(x, t) \mathrm{d} x\right)^{2} .
$$

As we can see, Proposition 1.1 plays an essential role in obtaining the above inequality, which is the key estimate in our analysis of the finite time singularity of the nonlocal system.

By the definition of $\phi$, we have the following inequality:

$$
\int_{a}^{b} u^{2}(x, t) d x \geq \frac{1}{b-a} \int_{a}^{b} \phi(x) u^{2}(x, t) \mathrm{d} x .
$$

Combining (36) with (37), we obtain the following key estimate:

$$
\frac{d^{2}}{d t^{2}} \int_{a}^{b} \phi(x) u^{2}(x, t) \mathrm{d} x \geq \frac{2}{\pi(b-a)^{2}}\left(\int_{a}^{b} \phi(x) u^{2}(x, t) \mathrm{d} x\right)^{2}
$$

Denoting $F(t)=\int_{a}^{b} \phi(x) u^{2}(x, t) \mathrm{d} x$ we obtain the ODE inequality system

$$
F_{t t} \geq \frac{2}{\pi(b-a)^{2}} F^{2}, \quad F_{t}(0)=C>0, \quad F(0)=\int_{a}^{b} \phi u_{0}^{2}>0 .
$$

Since $F_{t}(0)=C>0$, integrating (39) from 0 to $t$ gives $F_{t}(t)>0$ for all $t \geq 0$. Denote $\widetilde{F}(t) \equiv F(t)-F(0)$. Then we have $\widetilde{F}(t) \geq 0$ for $t \geq 0, \widetilde{F}_{t}>0$ and $\widetilde{F}(0)=0$. Since $F(0)>0$ and $\widetilde{F}(t) \geq 0$, it is easy to show that $\widetilde{F}$ satisfies the same differential inequality (39) as $F$. Therefore we can set $F(0)=0$ in the following analysis without loss of generality.

Multiplying $F_{t}$ to $F_{t t} \geq \frac{2}{\pi(b-a)^{2}} F^{2}$ and integrating in time, we obtain

$$
\frac{d F}{d t} \geq \sqrt{\frac{4}{3 \pi(b-a)^{2}} F^{3}+C^{2}}
$$

It is easy to see from the above inequality that $F$ must blow up in a finite time. Define

$$
I(x)=\int_{0}^{x} \frac{\mathrm{d} y}{\sqrt{y^{3}+1}}, \quad J=\left(\frac{3 \pi(b-a)^{2} C^{2}}{4}\right)^{1 / 3} .
$$

Integrating (40) in time gives

$$
I\left(\frac{F(t)}{J}\right) \geq \frac{C t}{J}
$$


Observe that both $I$ and $F$ are strictly increasing functions, and $I(x)$ is bounded for all $x>0$ while the right hand side of (41) increases linearly in time. It follows from (41) that $F(t)$ must blow up no later than

$$
T^{*}=\frac{J}{C} I_{\infty}=\left(\frac{4 C}{3 \pi(b-a)^{2}}\right)^{-1 / 3} I_{\infty}
$$

This contradicts with the assumption that the nonlocal system has a globally smooth solution for the given initial condition $u_{0}$ and $v_{0}$. This contradiction implies that the solution of the nonlocal system (4)-(5) must develop a finite time singularity in the $H^{1}$ norm no later than $T^{*}$ given by (42). This completes our proof of Theorem 4.1.

\subsection{Periodic Initial Data}

In this subsection, we will extend the analysis of finite time singularity formation of the nonlocal system to periodic initial data. Below we state our main result:

Theorem 4.2 We assume that the initial values $u_{0}, v_{0}$ are periodic functions with period $2 \pi$ and the support of $u_{0}$ is contained in $(a, b) \subset(0,2 \pi)$ with $b-a<\pi$. Moreover, we assume that $u_{0}, v_{0} \in H^{1}[0,2 \pi]$. Let $\phi(x)$ be a $2 \pi$-periodic Lipschitz continuous function with $\phi(x)=x-a$ on $[a, b]$, and

$$
C=4 \int_{a}^{b} \phi(x) u_{0}^{2} v_{0} \mathrm{~d} x, \quad I_{\infty}=\int_{0}^{+\infty} \frac{\mathrm{d} y}{\sqrt{y^{3}+1}} .
$$

If $C>0$, then the solution of the nonlocal system (4)-(5) must develop a finite time singularity in the $H^{1}$ norm no later than $T^{*}=\left(\frac{4 C \cos \left(\frac{b-a}{2}\right)}{3 \pi(b-a)^{2}}\right)^{-1 / 3} I_{\infty}$.

Proof As in the proof of Theorem 4.1, we also prove this theorem by contradiction. Assume that the nonlocal system with the given initial condition $u_{0}$ and $v_{0}$ has a globally smooth solution in $H^{1}$. As before, by differentiating (4) with respect to $t$, we obtain the following equation:

$$
\left(u^{2}\right)_{t t}=4\left(u_{t}\right)^{2}+4 u^{2} H\left(u^{2}\right) .
$$

Multiplying $\phi(x)$ to both sides of the above equation, integrating over $[0,2 \pi]$ and using Proposition 2.1, we obtain the following estimate:

$$
\begin{aligned}
\frac{d^{2}}{d t^{2}} \int_{a}^{b} \phi(x) u^{2}(x, t) \mathrm{d} x & =\frac{d^{2}}{d t^{2}} \int_{0}^{2 \pi} \phi(x) u^{2}(x, t) \mathrm{d} x \\
& =4 \int_{0}^{2 \pi} \phi(x)\left(u_{t}\right)^{2} \mathrm{~d} x+4 \int_{0}^{2 \pi} \phi(x) u^{2} H\left(u^{2}\right) \mathrm{d} x \\
& \geq 4 \int_{0}^{2 \pi} \phi(x) u^{2} H\left(u^{2}\right) \mathrm{d} x
\end{aligned}
$$




$$
\begin{aligned}
& =\frac{1}{\pi} \int_{0}^{2 \pi} \int_{0}^{2 \pi} u^{2}(x, t) u^{2}(y, t)(\phi(x)-\phi(y)) \cot \left(\frac{x-y}{2}\right) \mathrm{d} y \mathrm{~d} x \\
& =\frac{1}{\pi} \int_{a}^{b} \int_{a}^{b} u^{2}(x, t) u^{2}(y, t)(x-y) \cot \left(\frac{x-y}{2}\right) \mathrm{d} y \mathrm{~d} x \\
& \geq \frac{M}{\pi}\left(\int_{a}^{b} u^{2}(x, t) \mathrm{d} x\right)^{2},
\end{aligned}
$$

where $M=\min _{-(b-a) \leq x \leq b-a} x \cot (x / 2)$. Since $b-a<\pi$, we have

$$
M=\min _{-(b-a) \leq x \leq b-a} \frac{x \cos (x / 2)}{\sin (x / 2)} \geq \min _{-(b-a) \leq x \leq b-a} 2 \cos (x / 2)=2 \cos \left(\frac{b-a}{2}\right)>0 .
$$

Now, following the same procedure as in the proof of Theorem 4.1, we conclude that the solution must blow up no later than

$$
T^{*}=\left(\frac{4 M C}{6 \pi(b-a)^{2}}\right)^{-1 / 3} I_{\infty} \leq\left(\frac{4 C \cos \frac{b-a}{2}}{3 \pi(b-a)^{2}}\right)^{-1 / 3} I_{\infty}
$$

This contradicts with the assumption that the nonlocal system with the given initial condition $u_{0}$ and $v_{0}$ has a globally smooth solution. This contradiction implies that the solution of the nonlocal system (4)-(5) must develop a finite time singularity in the $H^{1}$ norm no later than $T^{*}$ given by (46). This completes the proof of Theorem 4.2.

Remark 4.1 We can also prove the finite time blowup of a variant of our nonlocal system

$$
u_{t}=2 u v, \quad v_{t}=-H\left(u^{2}\right)
$$

by choosing the test function $\phi(x)=b-x$. It is interesting to note that while the solution of (4) produces traveling waves that propagate to the right, the solution of (47) produces traveling waves that propagate to the left.

Remark 4.2 Our singularity analysis can be generalized to give another proof of finite time singularity formation of the Constantin-Lax-Majda model without using the exact integrability of the model. More precisely, we consider the Constantin-Lax-Majda model:

$$
\left\{\begin{array}{l}
u_{t}=u H(u) \\
u(t=0)=u_{0}(x), \quad x \in \Omega .
\end{array}\right.
$$

By choosing $\phi(x)=x-a$ and following the same procedure as in the proof of Theorem 4.1, we can show that if $u_{0}$ is smooth and has compact support, supp $u_{0}=[a, b]$ and $u_{0}(x)>0$ on $(a, b)$, then the $L_{1}$ norm of the solution of (48) must blows up no later than

$$
T^{*}=\frac{2 \pi(b-a)^{2}}{\int_{a}^{b} \phi(x) u_{0} \mathrm{~d} x}
$$


Below we will give a different and simpler proof of the finite time blowup for the ConstantinLax-Majda model.

Multiplying $\phi(x)$ to both sides of equation (48), integrating over the support $(a, b)$, and using Proposition 1.1, we obtain

$$
\frac{\mathrm{d}}{\mathrm{d} t} \int_{a}^{b}(x-a) u \mathrm{~d} x=\int_{a}^{b}(x-a) u H(u) \mathrm{d} x=\frac{1}{2 \pi}\left(\int_{a}^{b} u \mathrm{~d} x\right)^{2} .
$$

As $\int_{a}^{b}(x-a) u \mathrm{~d} x \leq(b-a) \int_{a}^{b} u \mathrm{~d} x$ due to $u \geq 0$ for $x \in[a, b]$, setting $F(t)=\int_{a}^{b}(x-a) u \mathrm{~d} x$ we have

$$
F_{t} \geq \frac{1}{2 \pi(b-a)^{2}} F^{2}, \quad F(0)=\int_{a}^{b} \phi(x) u_{0} \mathrm{~d} x>0 .
$$

This leads to

$$
F(t) \geq \frac{F(0)}{1-t F(0) / 2 \pi(b-a)^{2}},
$$

which implies the finite-time blowup of $F$ no later than $T^{*}=\frac{2 \pi(b-a)^{2}}{\int_{a}^{b} \phi(x) u_{0} \mathrm{~d} x}$.

Similar result can be obtained for periodic initial data following the same analysis of Theorem 4.2.

\section{Global regularity for a special class of initial data}

In this section, we will prove the global regularity of the solution of our nonlocal system for a special class of initial data. Below we state our main result in this section.

Theorem 5.1 Assume that $u_{0}, v_{0} \in H^{1}$. Further we assume that $u_{0}$ has compact support in an interval of size $\delta$ and $v_{0}$ satisfies the condition $v_{0} \leq-3$ on this interval. Then the $H^{1}$ norm of the solution of the nonlocal system (4)-(5) remains bounded for all time as long as the following holds

$$
\delta^{1 / 2}\left(\left\|v_{0 x}\right\|_{L^{2}}+\frac{1}{3} \delta^{1 / 2}\left\|u_{0 x}\right\|_{L^{2}}^{2}\right)<\frac{1}{4} .
$$

Moreover, we have $\|u\|_{L^{\infty}} \leq C e^{-3 t},\|u\|_{H^{1}} \leq C e^{-3 t}$, and $\|v\|_{H^{1}} \leq C$ for some constant $C$ which depends on $u_{0}, v_{0}$, and $\delta$ only.

Proof Note that (53) implies that $\delta^{1 / 2}\left\|v_{0 x}\right\|_{L^{2}}<\frac{1}{4}$ which gives $-4+2 \delta^{1 / 2}\left\|v_{0 x}\right\|_{L^{2}}<$ -3.5. By using an argument similar to the local well-posedness analysis, we can show that there exists $T_{0}>0$ such that $\|u\|_{H^{1}}$ and $\|v\|_{H^{1}}$ are bounded, $v<-2$ on $\operatorname{supp}(u)$, and $2 \delta^{1 / 2}\left\|v_{x}\right\|_{L^{2}}<1$ for $0 \leq t<T_{0}$.

Let $[0, T)$ be the largest time interval on which $\|u\|_{H^{1}}$ and $\|v\|_{H^{1}}$ are bounded, and both of the following inequalities hold:

$$
v<-2 \quad \text { on } \operatorname{supp}(u) \text { and } 2 \delta^{1 / 2}\left\|v_{x}\right\|_{L^{2}}<1 .
$$

We will show that $T=\infty$. 
We have for $0 \leq t<T$ that

$$
\frac{\mathrm{d}}{\mathrm{d} t} \int u_{x}^{2} \mathrm{~d} x=4 \int\left(v u_{x}^{2}+u v_{x} u_{x}\right) \mathrm{d} x \leq-8 \int u_{x}^{2} \mathrm{~d} x+4\|u\|_{L^{\infty}}\left\|v_{x}\right\|_{L^{2}}\left\|u_{x}\right\|_{L^{2}} .
$$

Observe that $\operatorname{supp}(u)=\operatorname{supp}\left(u_{0}\right)$ for all times. Let $\Omega=\operatorname{supp}\left(u_{0}\right)$. Since $\operatorname{supp}(u)$ has length $\delta$, we can use the Poincaré inequality to get

$$
\|u\|_{L^{\infty}} \leq \delta^{1 / 2}\left\|u_{x}\right\|_{L^{2}(\Omega)}=\delta^{1 / 2}\left\|u_{x}\right\|_{L^{2}}
$$

Therefore we obtain the following estimate:

$$
\begin{aligned}
\frac{\mathrm{d}}{\mathrm{d} t}\left\|u_{x}\right\|_{L^{2}} & \leq-4\left\|u_{x}\right\|_{L^{2}}+2 \delta^{1 / 2}\left\|v_{x}\right\|_{L^{2}}\left\|u_{x}\right\|_{L^{2}} \\
& =\left(-4+2 \delta^{1 / 2}\left\|v_{x}\right\|_{L^{2}}\right)\left\|u_{x}\right\|_{L^{2}}<-3\left\|u_{x}\right\|_{L^{2}} .
\end{aligned}
$$

Thus we have for $0 \leq t<T$ that

$$
\left\|u_{x}\right\|_{L^{2}} \leq\left\|u_{0 x}\right\|_{L^{2}} e^{-3 t}
$$

On the other hand, we have that

$$
\begin{aligned}
\frac{\mathrm{d}}{\mathrm{d} t} \int v_{x}^{2} \mathrm{~d} x & =4 \int v_{x} H\left(u u_{x}\right) \mathrm{d} x \leq 4\left\|v_{x}\right\|_{L^{2}}\left\|u u_{x}\right\|_{L^{2}} \\
& \leq 4\|u\|_{L^{\infty}}\left\|v_{x}\right\|_{L^{2}}\left\|u_{x}\right\|_{L^{2}} \leq 4 \delta^{1 / 2}\left\|v_{x}\right\|_{L^{2}}\left\|u_{x}\right\|_{L^{2}}^{2},
\end{aligned}
$$

where we have used the property that $\|H(f)\|_{L^{2}} \leq\|f\|_{L^{2}}$ and the Poincare inequality (56). Now using (58), we get

$$
\frac{\mathrm{d}}{\mathrm{d} t}\left\|v_{x}\right\|_{L^{2}} \leq 2 \delta^{1 / 2}\left\|u_{x}\right\|_{L^{2}}^{2} \leq 2 \delta^{1 / 2}\left\|u_{0 x}\right\|_{L^{2}}^{2} e^{-6 t} .
$$

As a consequence, we obtain for $0 \leq t<T$ that

$$
\left\|v_{x}\right\|_{L^{2}} \leq\left\|v_{0 x}\right\|_{L^{2}}+\frac{1}{3} \delta^{1 / 2}\left\|u_{0 x}\right\|_{L^{2}}^{2} .
$$

Now observe that at the left end of the support of $u, v_{t}=H u^{2}$ is always negative. Since $v_{0} \leq-3$ on the support of $u$, we conclude that $v(x, t) \leq-3$ at the left end of the support of $u$ for all times. Now, we apply the Poincaré inequality in the support of $u$ and use (61) to obtain

$$
v \leq-3+\delta^{1 / 2}\left\|v_{x}\right\|_{L^{2}(\Omega)} \leq-3+\delta^{1 / 2}\left(\left\|v_{0 x}\right\|_{L^{2}}+\frac{1}{3} \delta^{1 / 2}\left\|u_{0 x}\right\|_{L^{2}}^{2}\right),
$$

on $\operatorname{supp}(u)$ for all $t \in[0, T)$.

Next, we perform $L^{2}$ estimates. We can easily show by using $v_{t}=H u^{2}$ that

$$
\frac{1}{2} \frac{d}{d t} \int v^{2} d x=\int v H u^{2} d x \leq\|v\|_{L^{2}}\left\|u^{2}\right\|_{L^{2}} \leq\|v\|_{L^{2}}\|u\|_{L^{\infty}}^{2} \delta^{1 / 2}
$$


which gives

$$
\frac{d}{d t}\|v\|_{L^{2}} \leq \delta^{1 / 2}\|u\|_{L^{\infty}}^{2}
$$

It follows from (56) and (58) that

$$
\|u\|_{L^{\infty}} \leq \delta^{1 / 2}\left\|u_{0 x}\right\|_{L^{2}} e^{-3 t}
$$

Therefore, we obtain

$$
\frac{d}{d t}\|v\|_{L^{2}} \leq \delta\left\|u_{0 x}\right\|_{L^{2}}^{2} e^{-6 t}
$$

which implies

$$
\|v\|_{L^{2}} \leq\left\|v_{0}\right\|_{L^{2}}+\frac{1}{6} \delta\left\|u_{0 x}\right\|_{L^{2}}^{2},
$$

for $0 \leq t<T$.

Similarly, using $v<-2$ on the support of $u$, we can easily show that

$$
\|u\|_{L^{2}} \leq\left\|u_{0}\right\|_{L^{2}} e^{-4 t}
$$

for $0 \leq t<T$.

To summarize, we have shown that $\|u\|_{H^{1}}$ and $\|v\|_{H^{1}}$ are uniformly bounded for $0 \leq$ $t<T$, and

$$
\left\|v_{x}\right\|_{L^{2}} \leq\left\|v_{0 x}\right\|_{L^{2}}+\frac{1}{3} \delta^{1 / 2}\left\|u_{0 x}\right\|_{L^{2}}^{2}
$$

and

$$
v \leq-3+\delta^{1 / 2}\left\|v_{x}\right\|_{L^{2}} \leq-3+\delta^{1 / 2}\left(\left\|v_{0 x}\right\|_{L^{2}}+\frac{1}{3} \delta^{1 / 2}\left\|u_{0 x}\right\|_{L^{2}}^{2}\right),
$$

on $\operatorname{supp}(u)$ for $0 \leq t<T$.

By our assumption on the initial data, we have

$$
\delta^{1 / 2}\left(\left\|v_{0 x}\right\|_{L^{2}}+\frac{1}{3} \delta^{1 / 2}\left\|u_{0 x}\right\|_{L^{2}}^{2}\right)<\frac{1}{4} .
$$

Therefore, we have proved that if

$$
v<-2 \text { on } \operatorname{supp}(u) \text { and } 2 \delta^{1 / 2}\left\|v_{x}\right\|_{L^{2}}<1,
$$

$0 \leq t<T$, then we actually have

$$
v \leq-2.75 \text { on } \operatorname{supp}(u) \quad \text { and } \quad 2 \delta^{1 / 2}\left\|v_{x}\right\|_{L^{2}}<0.5,
$$

$0 \leq t<T$. This implies that we can extend the time interval beyond $[0, T)$ so that $(69)$ is still valid. This contradicts the assumption that $[0, T)$ is the largest time interval on which (69) is valid. This contradiction shows that $T$ can not be a finite number, i.e. (69) is true for all times. This in turn implies that $\|u\|_{H^{1}}$ and $\|v\|_{H^{1}}$ are bounded for all times. Moreover, we have shown that both $\|u\|_{L^{\infty}}$ and $\|u\|_{H^{1}}$ decay exponentially fast in time and $\|v\|_{H^{1}}$ is bounded uniformly for all times (see (63), (58), (61), (64) and (65)). This proves Theorem 5.1. 


\section{$6 \quad$ Numerical Results}

In this section, we perform extensive numerical experiments to study the nature of the singularities of the nonlocal system. Our numerical results demonstrate convincingly that the nonlocal system develops asymptotically self-similar singularities in a finite time for both the inviscid and the viscous nonlocal systems.

\subsection{Set-up of the Problem}

In our numerical study, we use the following nonlocal system without the factor of 2 in front of the nonlinear term $u v$ in the $u$-equation ${ }^{3}$ :

$$
\begin{aligned}
& u_{t}=u v+\nu u_{x x} \\
& v_{t}=H\left(u^{2}\right)+\nu v_{x x} .
\end{aligned}
$$

We study the nonlocal system for two types of initial data. The first type of initial data has compact support. The second type of initial data is periodic. The nature of the singularities for these two types of initial data is very similar. In the case of periodic data, we can use FFT to compute the Hilbert transform. This enables us to perform our computations with a very high space resolution.

Below we describe the initial data that we will use in our numerical experiments. We choose three different initial conditions. The first initial condition has compact support which lies in $\Omega=[0.45,0.55]$ and $v_{0} \equiv 0$. Within the compact support $\Omega, u_{0}$ is given by

$$
\text { Initial Condition I: } \quad u_{0}=\exp \left(1-\left(1-\left(\frac{x-0.5}{0.05}\right)^{2}\right)^{-1}\right) \text { for } x \in \Omega, \quad v_{0}=0 .
$$

We call this Initial Condition I. The largest resolution we use for Initial Condition I is $N=16,384$. The timestep is chosen to be $\Delta t=10^{-3} /\|u\|_{L^{\infty}}$ in order to resolve the maximum growth of $\|u\|_{L^{\infty}}$.

The last two initial conditions are periodic with period one. They are given as follows:

$$
\begin{array}{lll}
\text { Initial Condition II: } & u_{0}=2+\sin (2 \pi x)+\cos (4 \pi x), & v_{0}=0, \\
\text { Initial Condition III: } & u_{0}=\frac{1}{1.2+\cos (2 \pi x)}, & v_{0}=0 .
\end{array}
$$

We call them Initial Condition II and Initial Condition III respectively. The largest resolution that we use for these two periodic initial conditions is $N=262,144=2^{18}$, and the timestep is chosen to be $\Delta t=10^{-3} /\|u\|_{L^{\infty}}$.

We use the fourth order classical Runge-Kutta method to discretize the inviscid nonlocal system in time. For the viscous nonlocal system, we consider only periodic initial data since the solution will not have compact support anymore. In order to remove the stiffness of the

\footnotetext{
${ }^{3}$ As we have shown in Section 2, dropping this factor only changes the scaling of the solution.
} 
time discretization due to the viscous term, we first apply Fourier transform to the nonlocal system to obtain

$$
\begin{aligned}
& \partial_{t} \widehat{u}(k, t)=\widehat{(u v)}(k, t)-\nu k^{2} \widehat{u}(k, t), \\
& \partial_{t} \widehat{v}(k, t)=-i \operatorname{sgn}(k) \widehat{\left(u^{2}\right)}(k, t)-\nu k^{2} \widehat{v}(k, t),
\end{aligned}
$$

where $\widehat{u}(k, t)$ is the Fourier transform of $u$ and $k$ is the wave number. We then reformulate the viscous term as an integral factor

$$
\begin{aligned}
\frac{\partial}{\partial t}\left(e^{\nu k^{2} t} \widehat{u}(k, t)\right) & =e^{\nu k^{2} t} \widehat{(u v)}(k, t), \\
\frac{\partial}{\partial t}\left(e^{\nu k^{2} t} \widehat{v}(k, t)\right) & =-i \operatorname{sgn}(k) e^{\nu k^{2} t} \widehat{\left(u^{2}\right)}(k, t) .
\end{aligned}
$$

Now we apply the classical Runge-Kutta method to discretize the above system in time. The resulting time discretization method will be free of the stiffness induced by the viscous term.

For periodic initial data, we use the spectral method to discretize the Hilbert transform by using the explicit formula $\widehat{H}(k)=-i \operatorname{sgn}(k)$. For initial data of compact support, we use the well-known alternating trapezoidal rule to discretize the Hilbert transform which gives spectral accuracy. For the sake of completeness, we describe the method below, see also [22]. Let $x_{j}=j h$ the grid point and $h>0$ is the grid size. The alternating trapezoidal rule discretization of the Hilbert transform is given by the following quadrature:

$$
H(f)\left(x_{i}\right)=\sum_{(j-i) o d d} \frac{f_{j}}{x_{i}-x_{j}} 2 h .
$$

Therefore, our numerical method has spectral accuracy in space and and fourth order accuracy in time. The high order accuracy of the method plus high space resolution and adaptive time-stepping is essential for us to resolve the asymptotically self-similar singular solution structure of the nonlocal system.

\subsection{Asymptotically self-similar blowup of the inviscid nonlocal system}

In the singularity analysis, we have proved that the nonlocal system must develop a finite time singularity for a large class of initial data. However, the singularity analysis does not tell us the nature of the singularity. Understanding the nature of the singularity is the main focus of our numerical study. Our numerical results show that for all three initial conditions we consider here, they develop asymptotically self-similar singularities in a finite time. The numerical evidence of self-similar singularities is quite convincing for all three initial data that we consider. As is the case for the original 3D model, the mechanism of forming such self-similar blowup of the nonlocal system is due to the fact that we neglect the convection term in our model. As it is demonstrated in $[15,16]$, the convection term tends to destroy the mechanism for generating the finite time blowup in the $1 \mathrm{D}$ or $3 \mathrm{D}$ model. Indeed, a recent numerical study shows that the 3D incompressible Euler equation does not seem to grow faster than double exponential in time $[13,12]$. 
We use the following asymptotic singularity form fit to predict the singularity time and the blowup rate:

$$
\|u\|_{L^{\infty}}=\frac{C}{(T-t)^{\alpha}}
$$

where $T$ is the blowup time. We find that near the singularity time, the inverse of $\|u\|_{L^{\infty}}$ is almost a perfect linear function of time, see Figure 1. To obtain a good estimate for the singularity time, we perform a least square fit for the inverse of $\|u\|_{L^{\infty}}$. We find that $\alpha=1$ gives the best fit. The same least square fit also determines the potential singularity time $T$ and the constant $C$.

To confirm that the above procedure indeed gives a good fit for the potential singularity, we plot $\|u\|_{\infty}^{-1}$ as a function of time with a sequence of increasing resolutions against the asymptotically form fit for the three initial conditions we consider here. In Figure 1, we perform such comparison for Initial Condition I with a sequence of increasing resolutions from $N=4096$ to $N=16384$. We can see that the agreement between the computed solutions and the asymptotically fitted solution is excellent as the time approaches the potential singularity time. In the lower box of Figure 1, we plot $\|u\|_{\infty}$ computed by our adaptive method against the form fit $C /(T-t)$ with $T=2.36752830915169$ and $C=1.67396437016231$. The computed solutions and the asymptotically fitted solution are almost indistinguishable. This asymptotic blowup rate is qualitatively similar to that of the 3D model [16].

We have also performed a similar comparison between the computed $\|u\|_{L^{\infty}}$ and the asymptotically fitted solution for Initial Conditions II and III in Figures 2 and Figures 3 respectively. For these two periodic initial conditions, we can afford even higher resolutions ranging from $N=2^{14}$ to $N=2^{18}$. Again, we observe excellent agreement between the computed solutions and the asymptotically fitted singular solution.

After we obtain an estimate for the singularity time, we can use it to look for a dynamically rescaled profile $U(\xi, t), V(\xi, t)$ near the singularity of the form

$$
\begin{array}{ll}
u(x, t)=\frac{1}{T-t} U\left(\frac{x-x_{0}(t)}{(T-t)^{\beta}}, t\right), \quad \text { as } t \rightarrow T, \\
v(x, t)=\frac{1}{T-t} V\left(\frac{x-x_{0}(t)}{(T-t)^{\beta}}, t\right), \quad \text { as } t \rightarrow T,
\end{array}
$$

where $T$ is the predicted blowup time in the singularity form fit (78), $\beta$ is a parameter to be determined, and $x_{0}(t)$ is the location in which $|u|$ achieves its global maximum at $t$.

Again, we use a least square fit to determine $\beta$. Our numerical study indicates that $\beta=\frac{1}{2}$ with a logarithmic correction. More precisely, we find that the dynamically rescaled variable $\xi$ has the form:

$$
\xi=\frac{x-x_{0}(t)}{(T-t)^{1 / 2} \log (1 /(T-t))^{1 / 2}} .
$$

In terms of this rescaling variable $\xi$, we define the dynamically rescaled profiles $U(\xi, t)$ and $V(\xi, t)$ through the following relationship:

$$
u(x, t)=\frac{1}{T-t} U(\xi, t)
$$



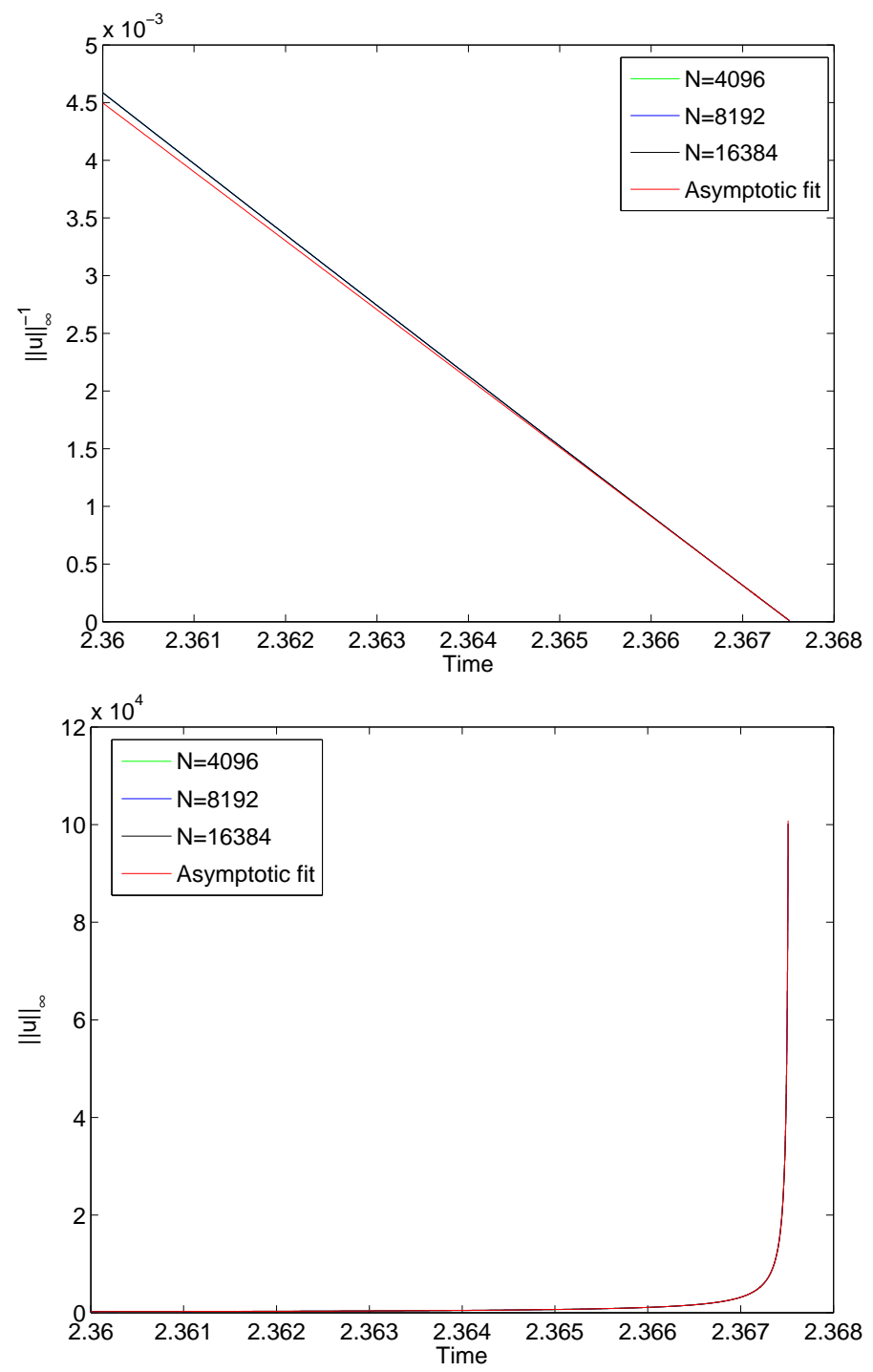

Figure 1: Top: The inverse of $\|u\|_{L^{\infty}}$ (black) versus the asymptotic fit (red) for Initial Condition I with $\nu=0$. The fitted blowup time is $T=2.36752830915169$ and the scaling constant is $C=1.67396437016231$. Bottom: $\|u\|_{L^{\infty}}$ (black) versus the asymptotic fit (red) for Initial Condition I. 


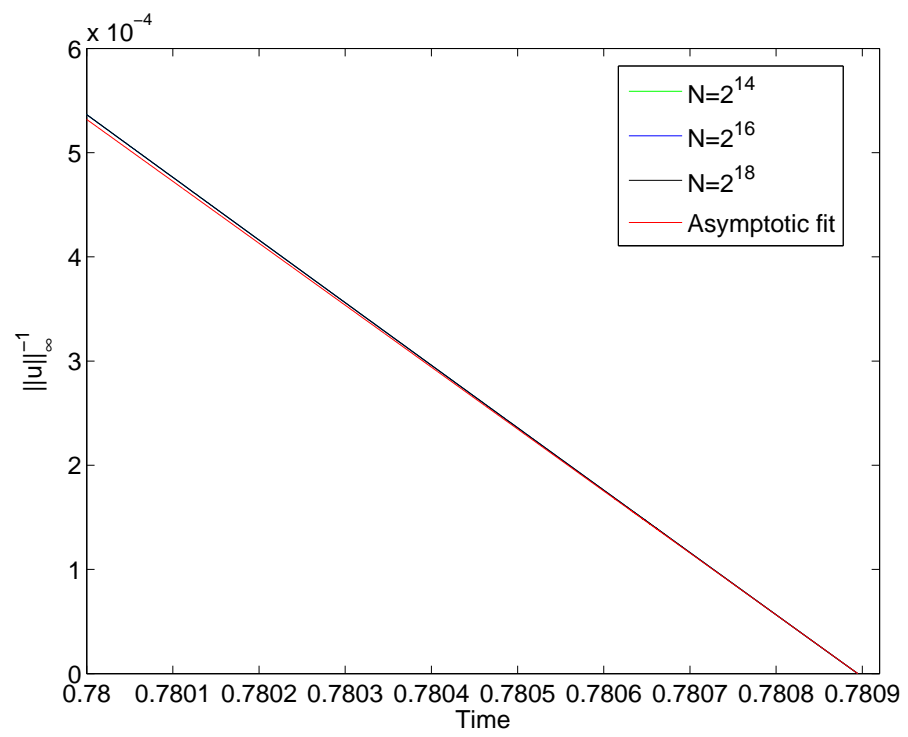

Figure 2: The inverse of $\|u\|_{L^{\infty}}$ (black) versus the asymptotic fit (red) for Initial Condition II with $\nu=0$. The fitted blowup time is $T=0.780894805082166$ and the scaling constant is $C=1.68253514799506$.

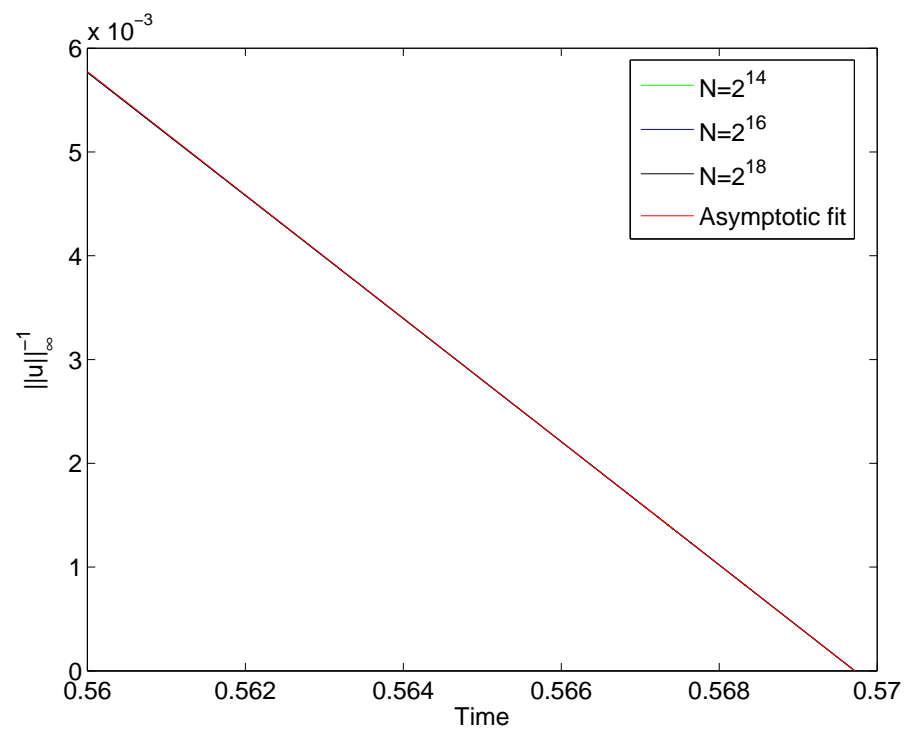

Figure 3: The inverse of $\|u\|_{L^{\infty}}$ (black) versus the asymptotic fit (red) for Initial Condition III with $\nu=0$. The fitted blowup time is $T=0.569719056780405$ and the scaling constant is $C=1.68293676812485$. 


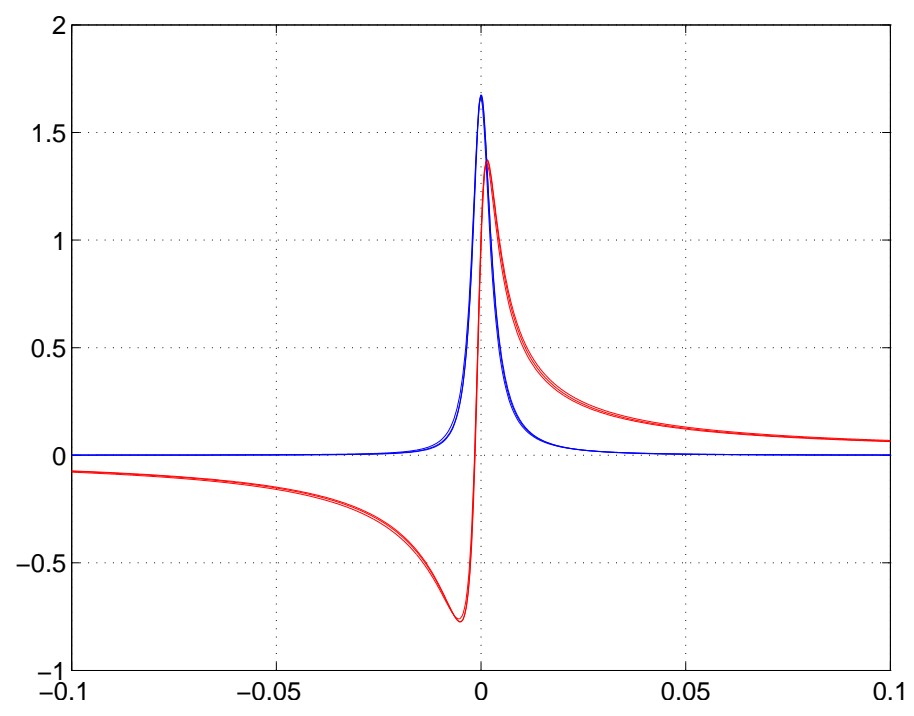

Figure 4: Rescaled profiles $U$ and $V$ for Initial Condition I with $\nu=0$ at three different times: $t=2.36710445318745,2.36743324526419$ and 2.36750705502071 , the corresponding maximum values of $u$ are 3948, 17617 and 78422 respectively. Blue: profile of $u$; Red: profile of $v$.

$$
v(x, t)=\frac{1}{T-t} V(\xi, t) .
$$

In Figure 4, we plot the self-similar profiles $U$ and $V$ at three different times for Initial Condition I. We can see that the rescaled profiles for these three different times agree with one another very well. From Figure 4, we can see that there is a significant overlap between the inner region of $U$ and the inner region of $V$ where $V$ is positive. Such overlap persists dynamically and is responsible for producing a quadratic nonlinearity in the right hand side of the $u$-equation, which has the form $2 u v$. On the other hand, we observe that the position at which $u$ achieves its global maximum is not in phase with the position at which $v$ achieves its global maximum. In fact, the positive part of $V$ always moves ahead of $U$. This is a consequence of the property of the Hilbert transform. As a result, the nonlinear interaction between $u$ and $v$ produces a traveling wave that moves to the right. Such phenomena seem quite generic. We observe the same phenomena for all three initial conditions for both the inviscid and the viscous models. This phenomenon is also qualitatively similar to that of the 3D model [16].

The strong alignment between the rescaled profile of $u$ and $v$ is the main mechanism for the solution of the nonlocal system to develop an asymptotically self-similar singularity in the form given by (81) and (82)-(83). We observe essentially the same phenomena for Initial Conditions II and III, see Figure 5.

It is interesting to see how the different rescaled profiles corresponding to different initial conditions are related to one another. In Figure 5 (top), we put three profiles from three different initial conditions together. The profile from Initial Condition III is the widest while 
the profile from Initial Condition II is narrower than that from Initial Condition III. The profile from Initial Condition I is the narrowest of the three initial conditions. But what is amazing is that they can match each other very well by rescaling the variable variable $\xi$. To match the three rescaled profiles, we keep the profile from Initial Condition III unchanged. In order to match the profile from Initial Condition III, we change the profile from Initial Condition II by rescaling $\xi \rightarrow \xi / 1.58$, and change the profile from Initial Condition I by rescaling $\xi \rightarrow \xi / 19.5$. As we can see from Figure 5 (bottom), the three rescaled profiles is almost indistinguishable.

To gain some insight into this phenomenon, we perform some analysis of the self-similar solutions. We assume that the self-similar profiles converge to a steady state as $t \rightarrow T$.

$$
\begin{gathered}
u(x, t) \rightarrow \frac{1}{T-t} U(\xi ; \lambda), \quad \text { as } t \rightarrow T \\
v(x, t) \rightarrow \frac{1}{T-t} V(\xi ; \lambda), \quad \text { as } t \rightarrow T,
\end{gathered}
$$

where $\lambda=\lim _{t \rightarrow T}\left((T-t)^{1 / 2} \frac{d}{d t} x_{0}(t)\right)$.

If we neglect the logarithmic correction in $\xi$ and substitute above equations into the nonlocal system, we obtain the equations for $U$ and $V$ as follows:

$$
\begin{aligned}
U+\beta \xi U_{\xi}-\lambda U_{\xi} & =U V, \\
V+\beta \xi V_{\xi}-\lambda V_{\xi} & =H\left(U^{2}\right) .
\end{aligned}
$$

Let $U_{1}(\xi), V_{1}(\xi)$ be the solution of the self-similar system (86), (87) corresponding to $\lambda=1$, then the solution for $\lambda \neq 1$ can be obtained by using the following rescaling of the self-similar variable $\xi$ :

$$
\begin{aligned}
& U(\xi ; \lambda)=U_{1}\left(\lambda^{-1} \xi\right) \\
& V(\xi ; \lambda)=V_{1}\left(\lambda^{-1} \xi\right) .
\end{aligned}
$$

The profiles that are obtained from different initial conditions have different $\lambda$, but they can match each other by rescaling $\xi$. This may explain why we can match different rescaled profiles corresponding to different initial conditions by rescaling $\xi$.

\subsection{Asymptotically self-similar blowup of the viscous nonlocal system}

In this subsection, we perform computations to investigate the finite time singularity of the viscous nonlocal system. In our computations, we choose the viscosity coefficient to be $\nu=0.001$. Notice that the solution of the viscous nonlocal system can not keep the compact support, so we only perform our numerical study for Initial Conditions II and III which are periodic. The computational settings are the same as those in the inviscid case.

We use the same asymptotic singularity form fit as in the inviscid model, i.e.

$$
\|u\|_{L^{\infty}}=\frac{C}{(T-t)^{\alpha}}
$$



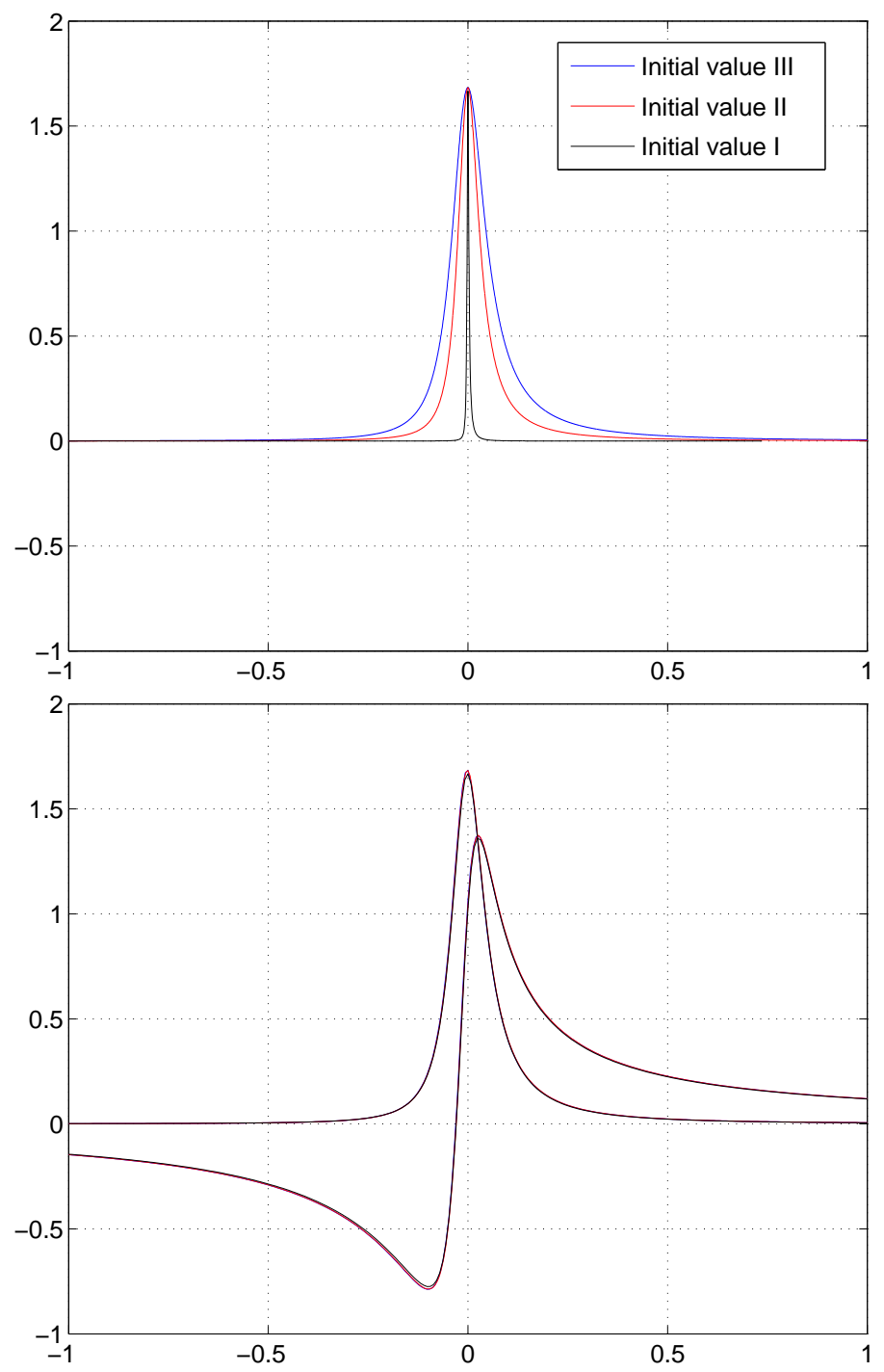

Figure 5: The self-similar profiles for Initial Conditions I-III respectively $(\nu=0)$. Top: The original profiles for $u$ for Initial Conditions I-III; Bottom: The rescaled profiles. Black: Initial Condition I; Red: Initial Condition II; Blue: Initial Condition III. 
where $T$ is the blowup time. In Figure 6 and Figure 7, we plot $\|u\|_{L^{\infty}}^{-1}$ versus the asymptotic singularity fit. We can see that as we increase resolutions from $N=2^{14}$ to $N=2^{18},\|u\|_{L^{\infty}}^{-1}$ converges to the asymptotic fit which is almost a perfect straight line. This suggests that $\alpha=1$. From these numerical results, we can see that adding viscosity with $\nu=0.001$ does not prevent the solution from blowing up and does not change the qualitative nature of the singular solution, although it postpones the blowup time.

Next, we study the rescaled profiles of the asymptotically self-similar solutions of the viscous nonlocal system. We look for a dynamically rescaled profile $U(\xi, t), V(\xi, t)$ near the singularity of the form

$$
\begin{array}{ll}
u(x, t)=\frac{1}{T-t} U\left(\frac{x-x_{0}(t)}{(T-t)^{\beta}}, t\right), & \text { as } t \rightarrow T, \\
v(x, t)=\frac{1}{T-t} V\left(\frac{x-x_{0}(t)}{(T-t)^{\beta}}, t\right), & \text { as } t \rightarrow T,
\end{array}
$$

where $T$ is the predicted blowup time in the singularity form fit (90), $\beta$ is a parameter to be determined, and $x_{0}(t)$ is the location in which $|u|$ achieves its global maximum at $t$. Again, we use a least square fit to determine $\beta$ and find that $\beta=1 / 2$ with a logarithmic correction. In Figures 8 and 9, we plot the rescaled profiles of the asymptotically self-similar solution for Initial Conditions II and III respectively. The dynamically rescaled variable $\xi$ has the same form as that of the inviscid nonlocal system, i.e.

$$
\xi=\frac{x-x_{0}(t)}{(T-t)^{1 / 2} \log (1 /(T-t))^{1 / 2}} .
$$

In Figure 8, we plot the self-similar profiles $U$ and $V$ at three different times for Initial Condition II. We can see that the rescaled profiles for these three different times agree with one another very well. As in the inviscid case, we observe that there is a significant overlap between the inner region of $U$ and the inner region of $V$ where $V$ is positive. Such overlap persists dynamically and is responsible for producing a quadratic nonlinearity in the right hand side of the $u$-equation. Similar observation can be made for the self-similar profiles for Initial Condition III, see Figure 9.

As we can see from Figures 8 and 9, the rescaled profiles of the viscous nonlocal system is qualitatively similar to those of the inviscid nonlocal systems. This is to be expected since there is a logarithmic correction in the rescaling variable $\xi$ in the inviscid nonlocal system. Consequently, the viscous term can not dominate the nonlinear term in the nonlocal system. On the other hand, we observe that the profiles corresponding to the viscous nonlocal system are wider and more symmetric than those corresponding to the inviscid nonlocal system. This seems to make sense because the viscosity tends to smooth the singularity and make the profiles smoother and more symmetric.

We have also performed a similar numerical study of the viscous nonlocal system with $\nu=0.01$ for Initial Conditions II and III. We find that the viscous nonlocal system develops an asymptotically self-similar singularity in a finite time with the same blowup rate and self-similar scaling as the case of $\nu=0.001$. 


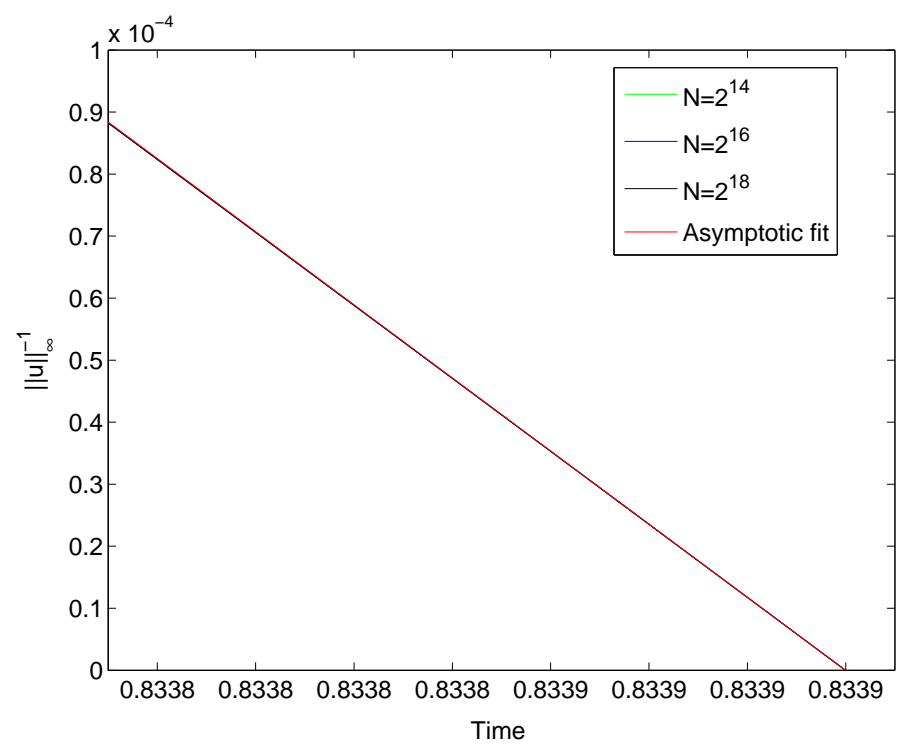

Figure 6: The inverse of $\|u\|_{\infty}$ (black) versus the asymptotic fit (red) for Initial Condition II with viscosity $\nu=0.001$. The fitted blowup time is $T=0.833919962702315$ and the scaling constant is $C=1.69630372479547$.

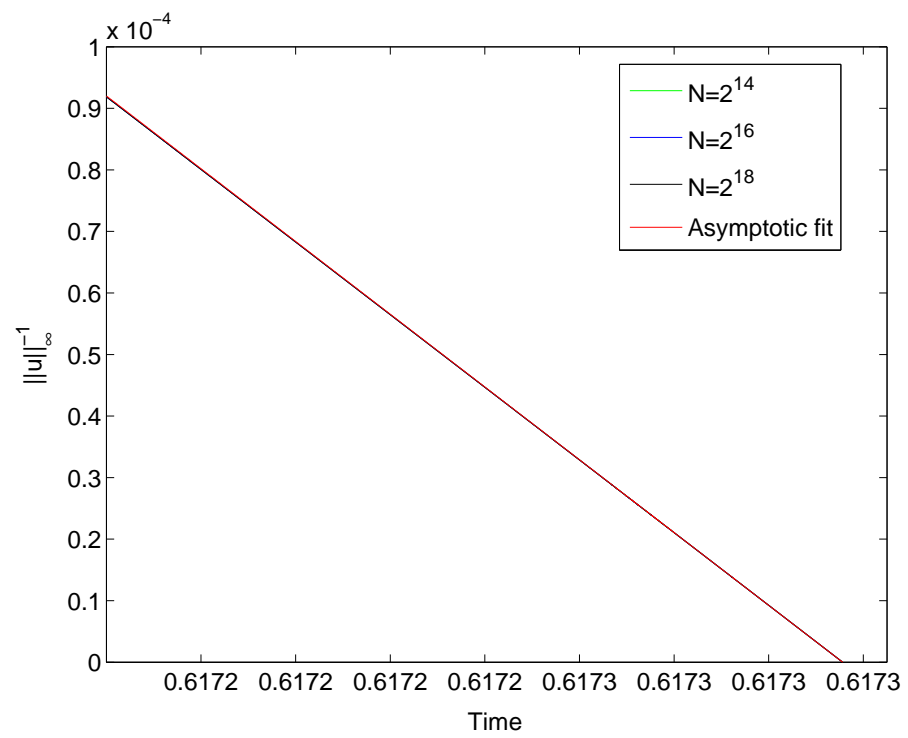

Figure 7: The inverse of $\|u\|_{\infty}$ (black) versus the asymptotic fit (red) for Initial Condition III with viscosity $\nu=0.001$. The fitted blowup time is $T=0.617315651741129$ and the scaling constant is $C=1.69150344092375$. 


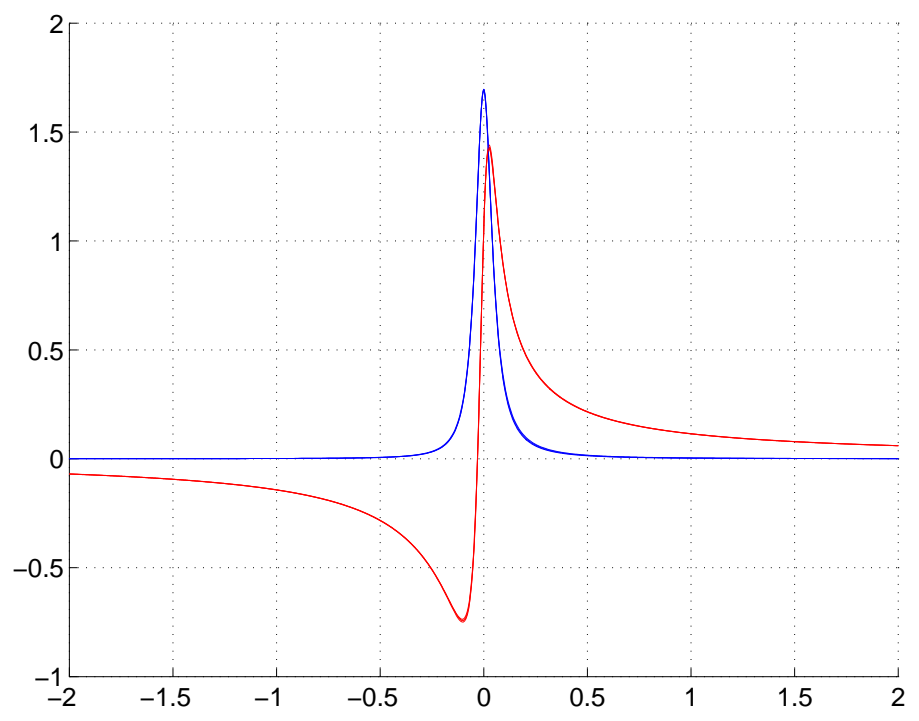

Figure 8: Rescaled profiles $U$ and $V$ for Initial Condition II with viscosity $\nu=0.001$ at $t=0.833917828434707,0.83391976141767$ and 0.833919943745501 respectively. The corresponding maximum values of u are 794399, 8416207 and 89496701 respectively. Blue: profile of $u$; Red: profile of $v$.

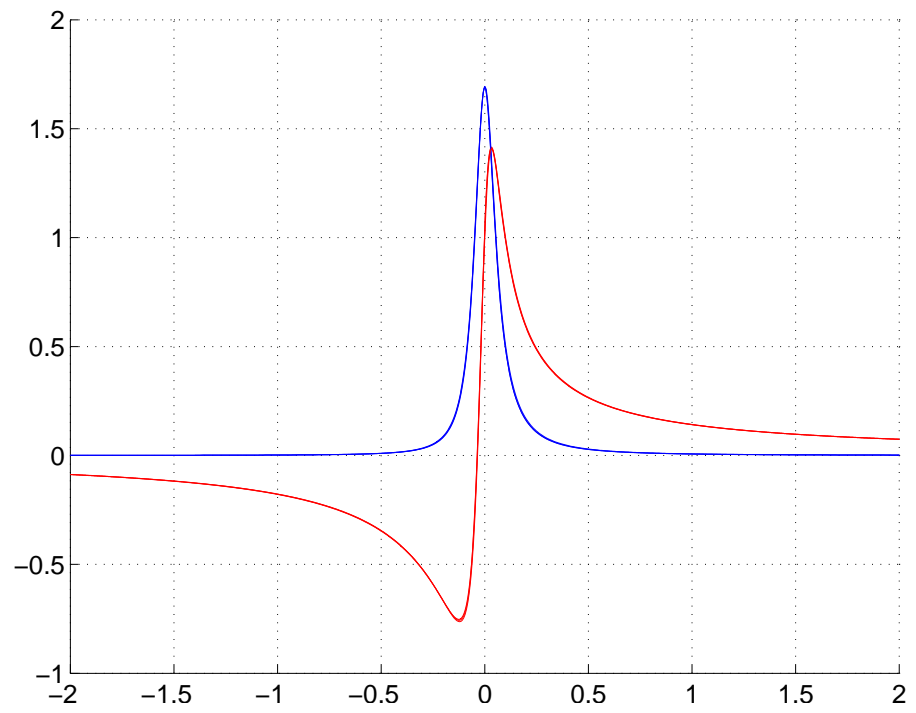

Figure 9: Rescaled profiles $U$ and $V$ for Initial Condition III with viscosity $\nu=0.001$ at $t=0.617313605456105,0.617315459830455$ and 0.617315633726175 respectively. The corresponding maximum values of $\mathrm{u}$ are 826395, 8808734 and 94072100 respectively. Blue: profile of $u$; Red: profile of $v$. 
Acknowledgments Dr. T. Hou would like to acknowledge NSF for their generous support through the Grants DMS-0713670 and DMS-0908546. The work of Drs. Z. Shi and S. Wang was supported in part by the NSF grant DMS-0713670. The research of Dr. C. Li was in part supported by the NSF grant DMS-0908546. The research of Dr. S. Wang was supported by the Grants NSFC 10771009 and phr-ihlb 200906103. The research of Dr. X. $\mathrm{Yu}$ was in part supported by the Faculty of Science start-up fund of University of Alberta, and the research grant from NSERC. This work was done during Drs. Li, Wang, and Yu's visit to ACM at Caltech. They would like to thank Prof. T. Hou and Caltech for their hospitality during their visit. Finally, we would like to thank the anonymous referee for the valuable comments and suggestions.

\section{References}

[1] J. T. Beale, T. Kato and A. Majda, Remarks on the breakdown of smooth solutions for the 3-D Euler equations. Comm. Math. Phys. 94 (1984), no. 1, 61-66.

[2] L. Caffarelli, R. Kohn and L. Nirenberg, Partial regularity of suitable weak solutions of the Navier-Stokes equations, Comm. Pure Appl. Math. 35 (1982), 771-831.

[3] A. P. Calderon and A Zygmund, On singular integrals. American J of Math., 78 (1956), no. 2, 289-309.

[4] D. Chae, A. Cordoba, D., Cordoba, and M. A. Fontelos, Finite time singularities in a $1 D$ model of the quasi-geostrophic equation, Adv. Math. 194 (2005), 203-223.

[5] P. Constantin, Note on loss of regularity for solutions of the 3D incompressible Euler and related equations, Commun. Math. Phys. 104 (1986), 311-326.

[6] P. Constantin, P. D. Lax and A. J. Majda, A simple one-dimensional model for the three-dimensional vorticity equation, Comm. Pure Appl. Math. 38 (1985), no. 6, 715724 .

[7] A. Cordoba, D., Cordoba, and M. A. Fontelos, Formation of singularities for a transport equation with nonlocal velocity, Adv. Math. 162(3) (2005), 1375-1387.

[8] S. De Gregorio, On a one-dimensional model for the 3-dimensional vorticity equation, J. Stat. Phys. 59 (1990), 1251-1263.

[9] S. De Gregorio, A partial differential equation arising in a $1 D$ model for the $3 D$ vorticity equation, Math. Method Appl. Sci. 19 (1996), no. 15, 1233-1255.

[10] J. Duoandikoetxea, The Hilbert transform and Hermite functions: A real variable proof of the $L^{2}$-isometry, J. Math. Anal. Appl. 347 (2008), 592-596.

[11] C. Fefferman, http://www.claymath.org/millennium/Navier-Stokes equations. 
[12] T. Y. Hou, Blow-up or no blow-up? A unified computational and analytic approach to study 3-D incompressible Euler and Navier-Stokes equations, Acta Numerica 18 (2009), 277-346. DOI: 10.1017/S0962492906420018.

[13] T. Y. Hou and R. Li, Dynamic depletion of vortex stretching and non-blowup of the 3-D incompressible Euler equations, J. Nonlinear Science 16 (2006), no. 6, 639-664.

[14] T. Y. Hou and Z. Lei, On partial regularity of a 3D model of Navier-Stokes equations, Commun. Math Phys. 287 (2009), no. 2, 589-612. DOI:10.1007/s00220-008-0689-9.

[15] T. Y. Hou and C. Li, Dynamic stability of the 3D axi-symmetric Navier-Stokes equations with swirl, Comm. Pure Appl. Math. 61 (2008), no. 5, 661-697.

[16] T. Y. Hou and Z. Lei, On the stabilizing effect of convection in 3D incompressible flows, Comm. Pure Appl. Math. 62 (2009), no. 4, 501-564. DOI: 10.1002/cpa.20254.

[17] T. Y. Hou, Z. Shi, and S. Wang, On singularity formation of a $3 D$ model for incompressible Navier-Stokes equations, arXiv:0912.1316v1 [math.AP], 2009.

[18] D. Li and J. Rodrigo, Blow up for the generalized surface quasi-geostrophic equation with supercritical dissipation, Comm. Math. Phys. 286(1) (2009), 111-124.

[19] D. Li and J. Rodrigo, On a one-dimensional nonlocal flux with fractional dissipation, preprint, 2009.

[20] A. J. Majda and A. L. Bertozzi, Vorticity and incompressible flow. Cambridge Texts in Applied Mathematics, 27. Cambridge University Press, Cambridge, 2002.

[21] H. Okamoto and K. Ohkitani, On the role of the convection term in the equations of motion of incompressible fluid, J. Phys. Soc. Japan 74 (2005), no. 10, 2737-2742.

[22] M. Shelley, A study of singularity formation in vortex sheet motion by a spectrally accurate vortex method, J. Fluid Mech. 244 (1992), 493-526.

[23] R. Temam, Navier-Stokes Equations. Second Edition, AMS Chelsea Publishing, Providence, RI, 2001. 